\title{
QUALITATIVE DISCRIMINATION BETWEEN PARACETAMOL TABLETS MADE BY NEAR INFRARED SPECTROSCOPY AND CHEMOMETRICS WITH REGARD TO POLYMORPHISM
}

\author{
(C) 2010 M. Khanmohammadi ${ }^{1 *}$, A.B. Garmarudi ${ }^{1,2}$, N. Moazzen $^{1}$, K. Ghasemi ${ }^{1}$ \\ ${ }^{1}$ Chemistry Department, Faculty of Science, IKIU, Qazvin, Iran \\ ${ }^{2}$ Department of Polymer \& Chemistry Laboratories, Engineering Research Institute, Tehran, Iran
}

Received March, 18, 2009

\begin{abstract}
Polymorphism is an important characteristic of pharmaceutical products because different polymorphs exhibit different physicochemical stabilities, dissolution rates, etc., which makes them different in therapeutic efficiency. Thus, it is important to control the polymorphic structure of pharmaceutical products. A spectroscopy method based on Fourier transform near infrared (FT-NIR) spectroscopy and chemometric techniques is introduced to classify paracetamol preparations according to polymorphic changes. X-ray diffraction (XRD) and FT-NIR studies were carried out on standard samples, paracetamol preparations (acetaminophen tablet), and also the additives. A direct comparison was performed between the spectroscopic data and those obtained by XRD. The NIR and XRD analyses of paracetamol preparations show some distinct differences, particularly in the Iranian tablet. These differences are found to be related to polymorphism and paracetamol purity. The cluster analysis (CA) and principal component analysis (PCA) were utilized to classify the paracetamol preparations. FT-NIR spectroscopy provides a simple, rapid and accurate qualitative analysis method for the identification of paracetamol polymorphs.
\end{abstract}

Ke y w o r d s: paracetamol, classification, polymorphism, chemometrics, Fourier transform, near infrared spectroscopy.

\section{INTRODUCTION}

As known, polymorphism is the ability of a solid material to exist in more than one form or crystal structure. It can also be a result of the existence of different conformers of the same molecule. Polymorphism is relevant to the development of pharmaceutical preparations. Many drugs get regulatory approval for only a single polymorph [1]. In the pharmaceutical industry, polymorphism and related phenomena are significant because they determine different properties (e.g. solubility, dissolution rate, chemical reactivity, melting point, resistance to degradation, bioavailability) of various forms of a substance, the properties that dictate the therapeutic efficiency. Therefore it is vital to control the polymorphs administered to patients and the conditions of the pharmaceutical product preparation. In order to control the quality of pharmaceutical products, the techniques that examine the crystal structure are required [ $2-6]$.

Polymorphic forms of a chemical can be discriminated by several techniques such as X-ray powder diffraction [ 7 ], differential scanning calorimetry (DSC) [8], thermal gravimetric analysis (TGA), microcalorimetry [9], vibrational spectroscopy, and dissolution kinetics [ 10-16].

Some of the foresaid techniques involve time consuming sample preparation or measurements, while some are not sufficiently accurate. Nowadays near infrared spectroscopy (NIR) has become a

\footnotetext{
*E-mail:mrkhanmohammadi@gmail.com
} 
Fig. 1. Structural formula of paracetamol destructive and easy to perform, with a simple sample preparation procedure. In the pharmaceutical industry, NIR spectroscopy has been used to determine various pharmaceutical characteristics. NIR spectra consist of overtones and combinations of fundamental vibrational bands in the mid-infrared region. Polymorphs have different IR spectra, and hence also different NIR spectra, because their molecular vibrational patterns are affected by differences in a specific crystal lattice.

A number of chemometric techniques have been employed in NIR quantitative and qualitative analysis since these approaches have been proved to be successful in extracting the desired information from unprocessed NIR spectra. Calibration methods such as multiple linear regressions (MLR), principal component analysis/principal component regression (PCA/PCR), partial least square regression (PLS) and pattern recognition methods are commonly used. NIR has been found to be a useful tool to ascertain whether a granulation process led to any changes in the crystal structure of the active material or not. PCA, SIMPLISMA, and orthogonal projection have been used to correct for temperature variation during the monitoring of polymorph conversion [6].

Paracetamol (4-acetamido phenol, Fig. 1) is an analgesic drug that is used worldwide in the production of tablets and other preparations. Paracetamol I is monoclinic, and paracetamol II is orthorhombic. Polymorph II can transform into polymorph I as the latter is more stable. In the crystalline state, the paracetamol molecules are linked by $\mathrm{NH} \ldots \mathrm{O}$ and $\mathrm{OH} \ldots \mathrm{O}$ hydrogen bonds into chains. The chains are further linked into two-dimensional networks, which in turn give 3-D layered structures with the van der Waals interactions between the layers. The chains are topologically the same in paracetamol I and paracetamol II, but the way how the chains are linked into the layers is different.

In this study, the CA and PCA techniques have been applied to classify paracetamol polymorphs in acetaminophen tablets, which are different in FT-NIR spectra depending on their crystalline form. The NIR spectroscopic data were consistent with those obtained by the congenital powder XRD method for the identification of the crystalline form.

\section{EXPERIMENTAL}

1. Apparatus and software. NIR spectra were recorded in reflectance mode using an ABBomem (MB 160D, Quebec, Canada) NIR instrument connected to a SpectraTech ${ }^{\circledR}$ microscope, in the $8000-4000 \mathrm{~cm}^{-1}$. The XRD technique was used as a reference method for the identification of the two crystalline forms of paracetamol. X-ray powder diffraction patterns were obtained using an X-ray diffractometer (XD-3A, Shimadzu, Japan). The measurement conditions were as follows: radiation source, $\mathrm{Cu} K_{\alpha}$; filter, $\mathrm{Ni}$; voltage $30 \mathrm{kV}$; current $5 \mathrm{~mA}$; receiving slit, $0.1 \mathrm{~mm}$; time constant, 1 second; scanning speed, $4^{\circ} 20 \mathrm{~min}^{-1}$. Cluster analysis and PCA chemometric methods were performed for the classification in MATLAB ${ }^{\circledR}$ ver. 7.1 media.

2. Material. Pharmaceutical preparations were from four different companies (Dolomol ${ }^{\circ}$, Tylenol $^{\odot}$, Panadol ${ }^{\odot}$, and Alborz Daru ${ }^{\odot}$ ) containing paracetamol as the principal active ingredient. These preparations are commercially available as tablets that contain talc powder, magnesium stearate, calcium phosphate, corn starch and polyvinyl pyrrolidone. The samples were from different batches of the product. The spectra of these tablets were recorded in NIR reflectance mode. Bulk paracetamol powders were from Hangzhou ${ }^{\circ}$, G.Amphray ${ }^{\circ}$, Anhui Chemicals ${ }^{\circ}$, and Alborz Daru ${ }^{\circ}$ Companies.

3. NIR spectroscopy and data analysis. All NIR studies were performed in the reflectance mode in the spectral region of 8000 to $4000 \mathrm{~cm}^{-1}$ by 32 scans and $8 \mathrm{~cm}^{-1}$ resolution. All spectra were baselined by the software. Hierarchical cluster analysis was used to classify the samples according to the crystalline form affecting the signals within the spectral region. Complete linkage and the absolute correlation coefficient were used to analyze the variance approach to evaluate the distance between clusters. In this process, the distance between all initial clusters is calculated. It was tried to perform the cluster analysis on similarities more than $99 \%$. PCA is probably the most widespread multivariate chemometric technique that has significantly affected the chemists' idea about "data analysis". Ac- 
cording to the importance of multivariate measurements in chemistry, PCA is one of the several multivariate methods that could be applied in classification. In PCA, it is tried to decompose the spectral data matrix $(\mathrm{X})$ to some components that are most effective in it. The scaling coefficient, which represents the structure of the samples, is called the score vector $(\mathrm{t})$; loading $(\mathrm{P})$ represents variations in the spectral features, and E corresponds to the residual spectral signals, e.g. noises. The equation below shows the relation between the data, score, and loading:

$$
\hat{\mathrm{X}}=\sum_{i=1}^{A} \mathrm{t}_{i} \mathrm{p}_{i}^{\mathrm{T}}+\mathrm{E}
$$

\section{RESULTS AND DISCUSSION}

1. Comparing the spectroscopic features. The production process of a pharmaceutical preparation consists of several steps, e.g. wet granulation, compression and/or addition of additives. Any of these steps may change the polymorphic state of the active principal ingredient. The monoclinic form of paracetamol has better medical properties than the orthorhombic form, and the pharmaceutical industry tries to monitor the pharmaceutical preparation to produce this particular crystalline form. The metastable orthorhombic form of paracetamol is prepared by melting and recrystallizing the commercially available monoclinic form. Fig. 2 shows the XRD pattern of four paracetamol bulk powder samples. The main XRD peaks of all samples were at $14^{\circ}, 15.9^{\circ}, 18^{\circ}, 19.4^{\circ}, 21.1^{\circ}, 21.9^{\circ}, 26.8^{\circ}, 27.3^{\circ}$, $28.3^{\circ}, 30.8^{\circ}$, and $31.6^{\circ}(2 \theta)$. These patterns were the same as a reference pattern of monoclinic crystalline paracetamol. The FT-NIR spectra of the paracetamol powders are shown in Fig. 3. According to Fig. 3, no diagnostically useful difference is observed in the FT-NIR spectra. It was important to investigate the probable interference of XRD signals from other ingredients with the paracetamol XRD signals. Cornstarch and PVP are organically-based compounds with no significant signal in their XRD patterns (Fig. 4). In addition, the main signals of other ingredients are at $22.1^{\circ}, 33.4^{\circ}$, and $57.2^{\circ}(2 \theta)$ for talc powder; at $24.8^{\circ}, 25.3^{\circ}, 25.5^{\circ}$, and $26.2^{\circ}$ (20) for magnesium stearate; and at $15.2^{\circ}$, $30.9^{\circ}, 35.2^{\circ}, 35.5^{\circ}, 36.2^{\circ}, 37.9^{\circ}$, and $38.3^{\circ}$

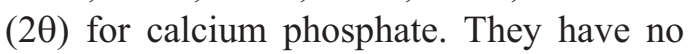
interference with the paracetamol pattern. Fig. 5 shows the XRD patterns of four preparation samples (acetaminophen tablets). The most important peaks are observed at $12.1^{\circ}, 13.8^{\circ}, 15.5^{\circ}, 16.7^{\circ}, 18.2^{\circ}$, $20.4^{\circ}, 23.4^{\circ}, 24.4^{\circ}$, and $26.5^{\circ}(2 \theta)$. The patterns for Alborz Daru ${ }^{\odot}$ and Dolomol ${ }^{\odot}$ samples show some dissimilarity in comparison with the others, while those of Panadol ${ }^{\odot}$ and Tylenol $^{\odot}$ are similar. The XRD results show that there are some significant differences between the Alborz Daru ${ }^{\circ}$ Iranian tablet and other acetaminophen tablets because an intense signal is observed in its XRD pattern at $18.5-22^{\circ}(2 \theta)$, which is not found in the XRD patterns of Panadol ${ }^{\circ}$ and Tyle$\mathrm{nol}^{\odot}$ tablets and is weakly pronounced in the XRD pattern of the Dolomol ${ }^{\odot}$ tablet. This may be attributed to the conversion of

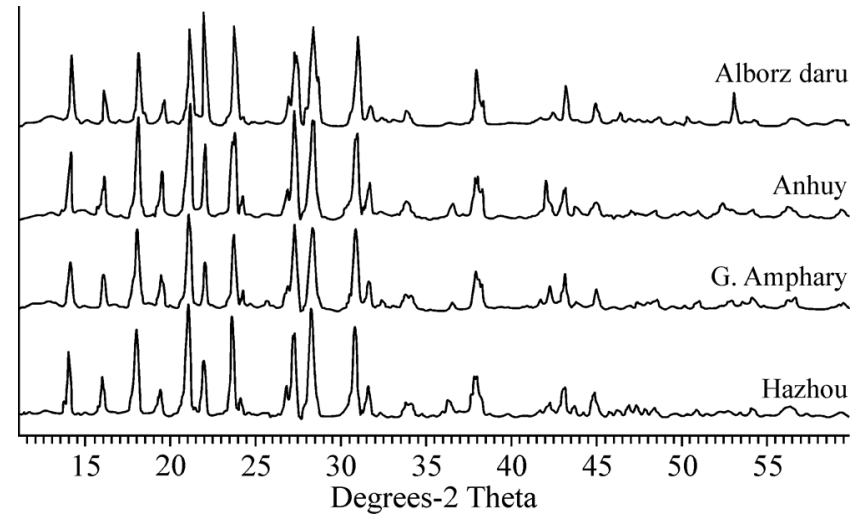

Fig. 2. XRD pattern of paracetamol bulk powder samples

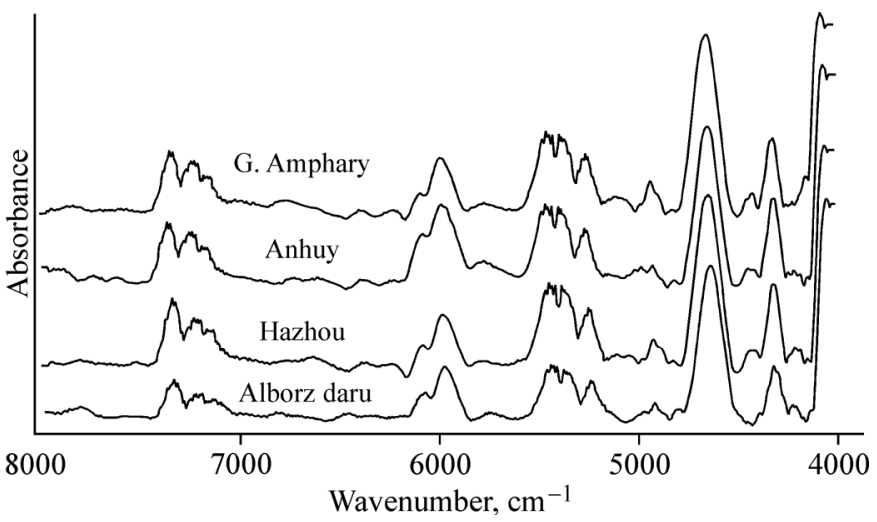

Fig. 3. FT-NIR spectra of paracetamol bulk powder samples 


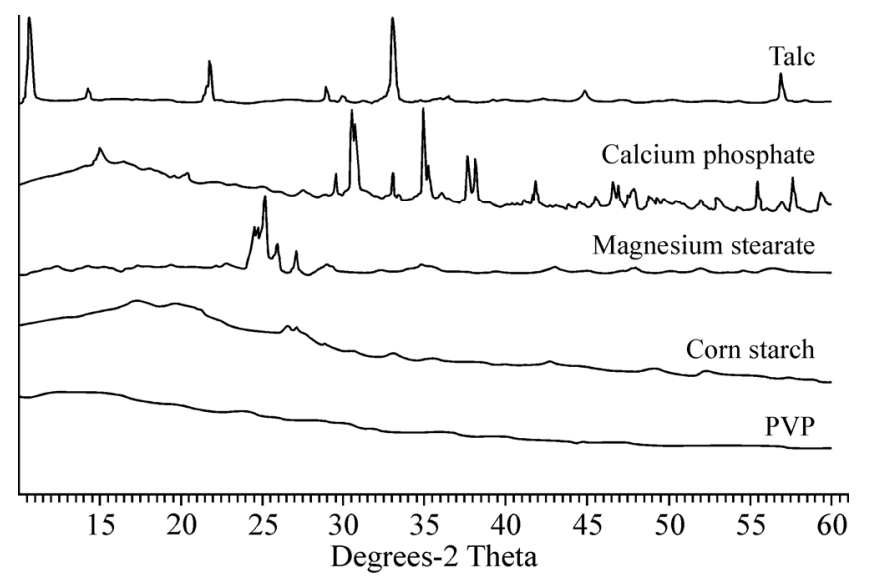

Fig. 4. XRD pattern of additives in the paracetamol tablet

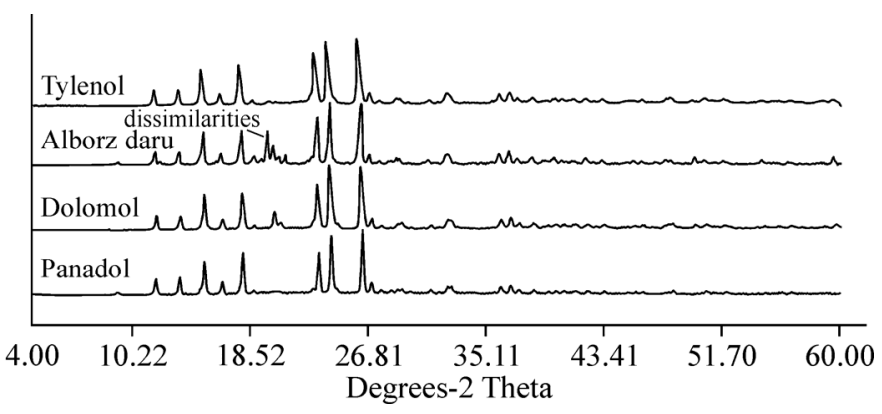

Fig. 5. XRD pattern of paracetamol tablets

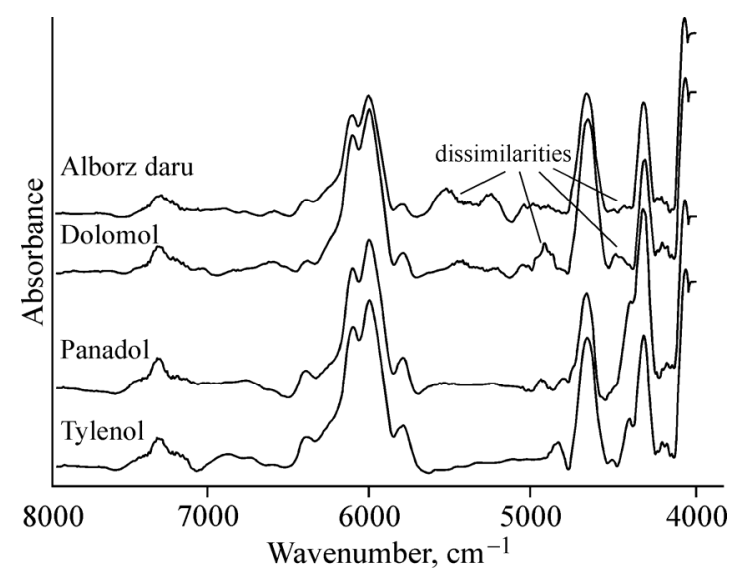

Fig. 6. FT-NIR spectra of paracetamol tablet samples

crystalline form. Fig. 6 shows the FT-NIR spectra of four paracetamol preparations. It was tried to investigate the probable differences utilizing the chemometric techniques in the NIR total spectral region, while the FT-NIR spectra of tablet additives show no important interference with the paracetamol signals.

2. Classification techniques. Cluster analysis methods are suitable for the detection of subgroups of entities. Other mathematical methods that can be used for this purpose are the correspondence analysis and the principal components analysis. For the classification of subjects, both of the latter two methods are appropriately used by reducing the number of dimensions to 2 , which afterwards can be judged simultaneously. In fact, this amounts to "clustering by eye". However, the disadvantage of this approach is a possibility that an existing grouping structure may be lost by projection. Cluster analysis is a popular technique whose basic objective is to discover sample groupings within the data. The technique is encountered in many fields, such as biology, geology, and geochemistry, under the names as unsupervised pattern recognition and numerical taxonomy. PCA is probably the oldest and best known of the techniques used for multivariate analysis. The overall goal of PCA is to reduce the dimensionality of a data set with simultaneous retaining of the information present in the data. Dimensionality reduction or data compression is possible with PCA because chemical data sets are often redundant.

Clustering methods attempt to find clusters of patterns (i.e. data points) in the measurement space, hence the term cluster analysis. Although there are several clustering algorithms used in classification methods e.g. K-means, K-median, Patrick-

Jarvis and fuzzy clustering varieties (FCV), it is worth noting that hierarchical clustering is the most widely used clustering algorithm $[6,18]$. The starting point for a hierarchical clustering experiment is the similarity matrix formed by first computing the distances between all pairs of points in the data set. Each distance is then converted into a similarity value

$$
S_{i k}=1-\frac{d_{i k}}{d_{\max }},
$$

where $S_{i k}$ (which varies from 0 to 1 ) is the similarity between samples $i$ and $k, d_{i k}$ is the Euclidean distance between samples $i$ and $k$, and $d_{\max }$ is the distance between the two most dissimilar samples (i.e. 
Fig. 7. Dendrogram of cluster analysis in the classification of paracetamol preparations

the largest distance) in the data set. There is a variety of methods to compute the distances between data points and clusters in hierarchical clustering; a practical method for this purpose was developed. In complete linkage, the distance between two clusters is the maximum distance between a variable in one cluster and a variable in another cluster. This is also called the furthest neighbor. In terms of the distance matrix,

$$
d_{\mathrm{mi}}=\max \left(d_{k j}-d_{i j}\right)
$$

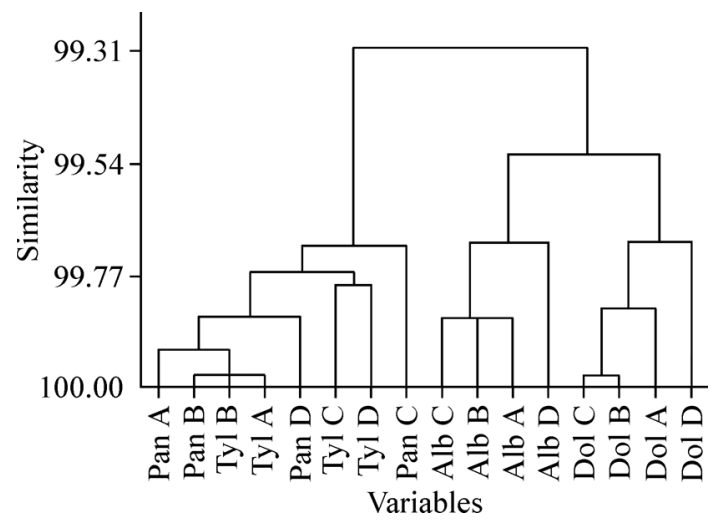

where the absolute correlation distance was applied to calculate the distance between the distances by

$$
d_{i j}=\left|1-\rho_{i j}\right|
$$

where $\rho_{i j}$ is the Pearson product moment correlation between variables $i$ and $j$. The final grouping of clusters (also called the final partition) is the grouping of clusters that will hopefully identify the groups whose observations or variables share common characteristics. The decision about final grouping is also called cutting the dendrogram (tree diagram), which is a graphical depiction of observations or variables combined into one cluster. It is important to know where to cut the dendrogram. At first, cluster analysis is executed without specifying a final partition. The similarity and distance levels are examined in the session window results and in the dendrogram. The similarity levels are observed by placing line over a horizontal line in the dendrogram. At any step the similarity level is the percent of the minimum distance relative to the maximum inter-observation distance in the data. The pattern of how similarity or distance values change from a step to step can help to choose the final grouping. The step where the values change abruptly can be a valid point for cutting the dendrogram, if this makes sense for the data.

In this study, the CA chemometric technique was used in the whole spectral region investigating four samples of each paracetamol preparation, and the dendrogram for 16 spectra of tablets was recorded. Fig. 7 shows $99 \%$ similarity on the dendrogram for the spectra, which assigns Dolomol ${ }^{\odot}$ and Alborz Daru ${ }^{\odot}$ tablets to one class, while Panadol $^{\odot}$ and Tylenol ${ }^{\odot}$ are in another class. The results are somehow similar to the XRD patterns. It is interesting that Alborz Daru tablets all fall in the same group and are different from the Dolomol samples. Evaluating the dendrogram, it is noted that this method is not a powerful tool for the diagnostics of Tylenol and Panadol tablets.

PCA creates "new" dimensions of the data [19] and evaluates a reduced number of independent principal components describing the information included in a system of characteristic but partly dependent variables. The aim of PCA is to find a few components that explain major variations within the data matrix. Each component in PCA is a weighted linear combination of the original variables. Only components with eigenvalues higher than the unity should be preferably considered [ $20-22]$.

In other words, PCA is an approach that can simplify the data interpretation by reducing the variable dimension of the $\mathrm{X}$ matrix down to a very small number of orthogonal principal components $\left(t_{1}\right.$, $\left.t_{2}, \ldots, t_{\mathrm{A}}\right)$ that summarize nearly all of the variance in the original data matrix. These new principal component variables ( $\left.t^{\prime} s\right)$ are simply linear combinations of the variables that explain the greatest variance, the next greatest variance, etc. That is the $\mathrm{X}_{(\mathrm{N} \times \mathrm{K})}$ matrix is decomposed as

$$
\mathrm{X}_{(\mathrm{N} \times \mathrm{K})}=\mathrm{T}_{(\mathrm{N} \times \mathrm{A})} \mathrm{P}^{\mathrm{T}}{ }_{(\mathrm{A} \times \mathrm{K})}+\mathrm{E} \text {, }
$$

where $\mathrm{T}$ is a matrix with only $\mathrm{A}(<\mathrm{N})$ columns containing the A new principal components variables $\left(t_{1}, t_{2}, \ldots, t_{\mathrm{A}}\right)$. The loading matrix $\mathrm{P}$ shows how these A principal components are related to the signal at the $\mathrm{K}$ wavenumbers, and each row of $\mathrm{P}^{\mathrm{T}}\left(p_{1}, p_{2}, \ldots, p_{\mathrm{A}}\right)^{\mathrm{T}}$ can be interpreted as a type of spectrum that defines the corresponding new variables $\left(t_{1}, t_{2}, \ldots, t_{\mathrm{A}}\right)$. PCA analysis by three essential factors was applied to the FT-NIR spectra. The 3D score plot of first scores (Fig. 8) shows that the tablets are clas- 


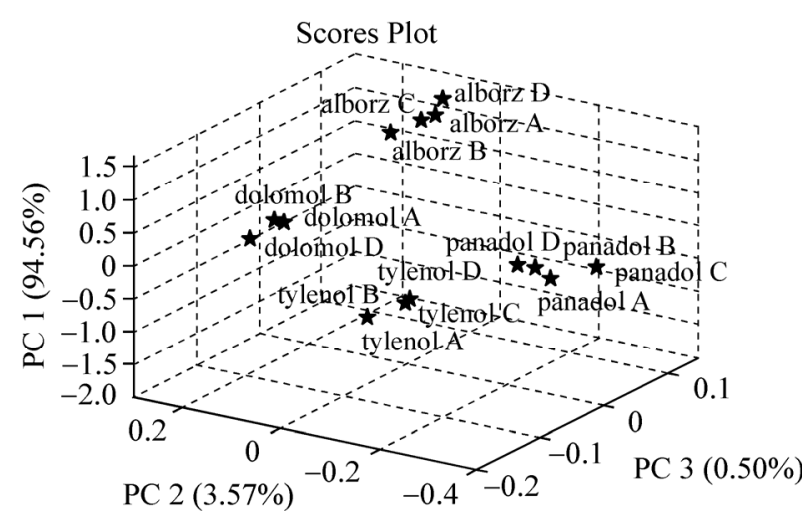

Fig. 8. Score plot of PCA for three PCs in the classification of paracetamol preparations

sified in separate groups. In other words, PCA could determine the different classes of tablet samples more accurately than CA.

\section{CONCLUSIONS}

The FT-NIR study in combination with chemometric techniques on the paracetamol polymorphs was demonstrated to have a significant advantage over the XRD method. This method is expected to provide a rapid qualitative analysis of polymorphs of preparations due to its simplicity, non-destructive nature, and sensitivity.

\section{REFERENCES}

1. Norris K., Aldridge P.K., Sekulic S.S. // Analyst. - 1997. - 122. - P. 549.

2. Britain H.G. Polymorphism in Pharmaceutical Solids, Marcel Dekker, New York, 1999.

3. Threlfall T.L. // Analyst. - 1995. - 120. - P. 2435.

4. Blanco M., Villar A. // Ibid. - 2000. - 125. - P. 2311.

5. Otsuka M., Kato F., Matsuda Y. // Ibid. - 2001. - 126. - P. 1578.

6. Otsuka M., Kato F., Matsuda Y. // AAPS Pharmsci. Teck. - 2000. - 2. - P. 9.

7. Yoshino H., Hagiwara Y., Kobayashi S., Samejima M. // Chem. Pharm. Bull. - 1984. - 32. - P. 1523.

8. Kaneniwa N., Otsuka M., Hayashi T. // Ibid. - 1985. - 33. - P. 3447.

9. Ahmed H., Buckton G., Rawlins D.A. // Int. J. Pharm. - 1996. - 130. - P. 195.

10. Taylor L.S., Zografi G. // Pharm. Res. - 1998. - 15. - P. 755.

11. Helmy R., Zhou G.X., Chen Y.W. et al. // Anal. Chem. - 2003. - 75. - P. 605.

12. Salari A., Young R.E. // Int. J. Pharm. - 1998. - 163. - P. 157.

13. Dohi K., Kaneko F., Kawaguchi T. // J. Cryst. Growth. - 2002. - 237. - P. 2227.

14. Aaltonen J., Rantanen J., Siiria S. et al. // Anal. Chem. - 2003. - 75. - P. 5267.

15. Agatonovic-Kustrin S., Rades T., Wu V. et al. // J. Pharm. Biomed. Anal. - 2001. - 25. - P. 741.

16. Pöllänen K., Häkkinen A., Reinikainen S. et al. // Ibid. - 2005. - 38. - P. 275 - 284.

17. Blanco M., Coello J., Iturriaga H. et al. // Analyst. - 1998. - 123. - P. 135.

18. Vogt W., Nagel D. // Clin. Chem. - 1992. - 38. - P. 182.

19. Cameán A.M., Moreno I., López-Artíguez M. et al. // Talanta. - 2001. - 54. - P. 53.

20. Flury B., Riedwyl H. Multivariate Statistics - A Practical Approach, Chapman \& Hall, London, 1988.

21. Beebe K.R., Pell R.J., Seasholtz M.B. Chemometrics: A Practical Guide. - Wiley, New York, 1998.

22. Henrion R., Henrion G. Multivariate Datenanalyse, Springer-Verlag, Heidelberg, 1995. 